\title{
The nucleolar size is associated to the methylation status of ribosomal DNA in breast carcinomas
}

\author{
Maria Giulia Bacalini ${ }^{1,2,3 \dagger}$, Annalisa Pacilli ${ }^{1,4 \dagger}$, Cristina Giuliani ${ }^{5}$, Marianna Penzo ${ }^{1}$, Davide Treré ${ }^{1}$, Chiara Pirazzini ${ }^{1,3}$, \\ Stefano Salvioli ${ }^{1,3}$, Claudio Franceschi ${ }^{1,2,3}$, Lorenzo Montanaro ${ }^{1 *}$ and Paolo Garagnani $i^{1,2,6^{*}}$
}

\begin{abstract}
Background: There is a body of evidence that shows a link between tumorigenesis and ribosome biogenesis. The precursor of mature 18S, $28 \mathrm{~S}$ and $5.8 \mathrm{~S}$ ribosomal RNAs is transcribed from the ribosomal DNA gene (rDNA), which exists as 300-400 copies in the human diploid genome. Approximately one half of these copies are epigenetically silenced, but the exact role of epigenetic regulation on ribosome biogenesis is not completely understood. In this study we analyzed the methylation profiles of the rDNA promoter and of the $5^{\prime}$ regions of $18 \mathrm{~S}$ and $28 \mathrm{~S}$ in breast cancer.
\end{abstract}

Methods: We analyzed rDNA methylation in 68 breast cancer tissues of which the normal counterpart was partially available (45/68 samples) using the MassARRAY EpiTYPER assay, a sensitive and quantitative method with single base resolution.

Results: We found that rDNA locus tended to be hypermethylated in tumor compared to matched normal breast tissues and that the DNA methylation level of several CpG units within the rDNA locus was associated to nuclear grade and to nucleolar size of tumor tissues. In addition we identified a subgroup of samples in which large nucleoli were associated with very limited or absent rDNA hypermethylation in tumor respect to matched normal tissue.

Conclusions: In conclusion, we suggest that rDNA is an important target of epigenetic regulation in breast tumors and that rDNA methylation level is associated to nucleolar size.

\section{Background}

Epigenetic regulation of ribosomal DNA (rDNA) locus has a pivotal role in orchestrating ribosome biogenesis. Human cells contain about 400 copies of the ribosomal RNA (rRNA) genes organized as tandem, head-to-tail repeats $[1,2]$, which are located in the fibrillar centers and the dense fibrillar component of the nucleolus [3]. Each unit is $\sim 43 \mathrm{~kb}$ long and includes the $47 \mathrm{~S}$ rRNA encoding sequence $(\sim 13 \mathrm{~kb})$ and a non-transcribed intergenic spacer $(\sim 30 \mathrm{~kb})$. In physiological conditions, around half of these copies is allelically inactivated through a combination of epigenetic mechanisms including late replication time [4], specific repression factors [5,6] and methylation of rDNA promoter. rDNA promoter includes a core

\footnotetext{
*Correspondence: Iorenzo.montanaro@unibo.it; paolo.garagnani2@unibo.it ${ }^{\dagger}$ Equal contributors

'Department of Experimental, Diagnostic and Specialty Medicine, University of Bologna, Bologna, Italy

${ }^{2}$ Personal Genomics S.r.l., Verona, Italy

Full list of author information is available at the end of the article
}

promoter region, extending from -50 to +20 in respect to the transcription starting site (TSS), and an upstream control element (UCE) at -200 in respect to TSS. In humans, but not in rodents, both the UCE and the core promoter are $\mathrm{CpG}$ rich regions, classifiable as $\mathrm{CpG}$ islands, which usually show a complex methylation pattern $[7,8]$ that can affect rRNA expression $[9,10]$.

Bisulfite sequencing of clonal rDNA promoters has been used to characterize rDNA methylation status in several pathological conditions. Hypermethylation of rDNA promoter was described in brain from Alzheimer's disease [11] and suicide subjects [12], while methylation levels of $18 \mathrm{~S}$ and $28 \mathrm{~S} 5$ ' regions were decreased in white blood cells from systemic lupus erythematosus subjects [13]. rDNA hypermethylation occurs during aging [14], and accordingly accelerated methylation of ribosomal regions was shown in fibroblasts from subjects affected by Werner syndrome [15]. The analysis of rDNA methylation in tumor samples appears to be in this context of extreme interest. 
Ribosome biogenesis is a limiting factor in sustaining the increased demand for protein synthesis, a prerequisite for cell growth and cell proliferation [16,17], and, as consequence, the rate of ribosome production is notably enhanced in cancer cells. rDNA promoter was found hypomethylated in respect to corresponding normal tissue in human hepatocellular carcinomas [7] but not in prostate cancer [18]. On the contrary, Yan and colleagues used methylation-sensitive Southern blotting to show increased rDNA methylation in patients with breast cancer compared to the normal control tissue; rDNA hypermethylation resulted also in association with specific tumor features such as the negativity of oestrogen receptors and poor tumor differentiation status [19].

In this study we analyzed methylation levels of three different regions within rDNA genes (the promoter and 5 ' regions of $18 \mathrm{~S}$ and $28 \mathrm{~S}$ sequences). In order to precisely define rDNA methylation profiles in breast cancer we used the MassARRAY EpiTYPER assay, a more sensitive and quantitative method compared to Southern blot and to clonal sequencing. Furthermore, we investigated a possible correlation between the methylation status of single CpG sites, ribosomal biogenesis and the available clinical and bio-pathological parameters in order to define its possible impact in the biological and clinical behavior of the tumors.

\section{Methods}

\section{Patient materials, characterization and total DNA} extraction

The study was approved by the St Orsola-Malpighi Hospital's ethical review board (approval number 75/2011/U/TESS). All volunteers provided written, informed consent. Sixty eight breast carcinomas were selected from a series of consecutive patients who had undergone surgical resection for primary breast carcinoma at the Surgical Department of the University of Bologna between 2005 and 2012, on the sole basis of frozen tissue availability. For forty five patients we collected both tumoral and non tumoral adjacent tissues (later on named normal tissue). Each patient's clinical information was recorded and correspondent tissue was histologically characterized by a team of clinical pathologists to define its bio-pathological features according to standard criteria for both clinical parameters and TNM (Tumour-Nodal-Metastasis) classification [20]. The expression of the oncosuppressor protein p53, Estrogen and Progesteron Receptors (ER and PR respectively) and proliferative markers ki67 was measured by experts after specific immunohistochemical (IHC) staining at the Operative Unit of Anatomy, Pathological Histology of the Sant'Orsola-Malpighi University Hospital in Bologna using NovoLinkTM Polymer Detection System (Novocastra Laboratories Ltd.) and following the manufacturer's instruction. For IHC analysis, the following mouse monoclonal primary antibodies were used: p53 (1:400, Novocastra); ER (1:450, DakoCytomation, Glostrup, Denmark); PR (1:400, Novocastra); ki67 (1:200, Novocastra). Silver staining of Nucleolar Organizer regions (AgNORs) was performed as described below. Specimen collection and tissue analyses were approved by the Bologna University Ethical Committee on human tissues research. Tissues were preserved at $-80^{\circ} \mathrm{C}$ until use. A piece of $60 \mathrm{mg}$ for each sample was minced in liquid nitrogen and then lysed for total DNA extraction using buffers provided with NucleoSpinTissue Columns kit (Macherey Nagel) and following the manufacturer's instructions.

\section{EpiTYPER assay for quantitative DNA methylation analysis} Quantitative DNA methylation analysis of rDNA locus was performed using the EpiTYPER assay (Sequenom). Briefly, $1000 \mathrm{ng}$ of DNA were bisulphite converted using the EZ96 DNAMethylation Kit (Zymo Research Corporation) as previously described [21]. $10 \mathrm{ng}$ of bisulphite-treated DNA were PCR-amplified using the following primers: Ribo forward: AGGAAGAGAGGTGTGTTTTGGGGTTGAT TAGAG; Ribo reverse: CAGTAATACGACTCACTAT AGGGAGAAGGCTAAAACCCAACCTCTCCAAC; $18 S$ forward: AGGAAGAGAGGTTTGTTGTTTTTTTTGG ATGTGG; $18 S$ reverse: CAGTAATACGACTCACTA TAGGGAGAAGGCTCCTTACCTACCTAATTAATCCT ACCAA; $28 S$ forward: AGGAAGAGAGGGTATTTAG TTTTAGATGGAGTTTATTATT; $28 S$ reverse: CAGTA ATACGACTCACTATAGGGAGAAGGCTAAAAAAA ACTAACCAAAATTCCC. For each gene, CpG sites with missing values in more than $20 \%$ of the samples were removed, as well as samples with missing values in more than $20 \%$ of CpG sites.

\section{Selective nucleolar staining}

Five-micron sections were processed to perform the silver staining to visualize the nucleolar organizer regions and the argyrophilic proteins according to the guidelines of the "International committee on AgNOR quantitation" [22]. Tissues were deparaffinized in xylene and rehydrated in decreasing concentrations of ethanol and distilled water. After antigen retrieval in citrate buffer $\mathrm{pH} 6.0$ at $120^{\circ} \mathrm{C}, 1 \mathrm{~atm}$ for 21 minutes, the sections were then incubated in silver nitrate solution in a dark for $13 \mathrm{~min}$ at $37^{\circ} \mathrm{C}$. The silver staining solution consisted of one part of silver nitrate (Diapath) and two parts of $2 \%$ gelatin (Sigma) in 1\% formic acid (Carlo Erba) solution. Ultra pure distilled water was used for preparation of all solutions. The sections were then washed in distilled water, dehydrated in graded alcohol and xylene and cover slipped. The tissue was then ready for counts. After silver-staining, the NORs can be easily identified as black dots exclusively localized throughout the nucleolar area. Silver stained section was examined through a light microscope using Image-Pro 
Plus6 software (Media Cybernetics). The morphometric analysis was performed on a cell by cell basis of at least 200 nuclei and the mean nucleolar area was calculated.

The best cutoff value for the nucleolar size variable was obtained by the receiver operating characteristic curve and corresponded to the value of $5 \mu^{2}$.

\section{Statistical analysis}

DNA methylation values resulting from EpiTYPER assay are reported as continuous values ranging from $0(0 \%$ of methylation) to 1 (100\% of methylation). All analyses were performed in $\mathrm{R} 2.14$. For continuous parameters, the following thresholds were used in ANOVA and chi-squared tests: age $>50$ years; diameter $>20 \mathrm{~mm}$; p $53>10 \%$ of positivity; Ki67 $>20 \%$ of positivity; nucleolar size $>5 \mu \mathrm{m}^{2}$. p-values $<0.05$ were regarded as statistically significant.

\section{Results}

\section{Characterization of rDNA target regions}

To profile the rDNA methylation status in breast cancer, genomic DNA was extracted from 68 breast carcinomas samples; for 45 of them, pair-matched normal tissues were available.

We used the MassARRAY EpiTYPER system to analyze the methylation status of three target regions (amplicons) in the rRNA gene (Figure 1): i) RiboPromoter, from position -186 to position +48 (respect to the transcription start site), including both the upstream and the core promoters of the gene; ii) $18 S$, from position + 2946 to position +3432 , encompassing the 5 '-sequence of the $18 \mathrm{~S}$ region; iii) $28 \mathrm{~S}$, from position +7297 to position +7579 , encompassing the 5 '-sequence of the $28 \mathrm{~S}$ region. The three selected regions partially overlap with those previously analyzed in other studies [13,23].

The EpiTYPER assay returns quantitative methylation estimates of single CpGs or of small groups of adjacent CpGs (CpG units) depending on the sequence context. Using this method, we measured methylation levels of $8 \mathrm{CpG}$ units (13 CpGs), $14 \mathrm{CpG}$ units (26 CpGs) and 10 CpG units (15 CpGs) in RiboPromoter, $18 S$ and $28 S$ target regions respectively. In RiboPromoter amplicon, 7 CpGs were in the UCE region, while the remaining 6 were in the core promoter. The CpG units RiboPromoter CpG_15.16 (UCE region) and 18S_CpG_6.7 did not pass quality controls and were removed from further analysis.

\section{Assessment of rDNA methylation in normal and tumor tissues}

We first considered the correlation between methylation values in the 45 samples for which both tumor and normal tissue were available (Figure 2A). As expected, most of the CpG units within the same target region showed high correlation. In addition, comparable high levels of correlation were detected also between CpG units in different amplicons, although they are several thousands of bases apart. Correlation levels were slightly but statistically significantly lower in tumor in respect to normal tissue (mean correlation values $=0.85$ and 0.87 for tumor and normal tissue respectively, paired t-test $\mathrm{p}$-value $=$ $\left.1.36 \times 10^{-10}\right)$.

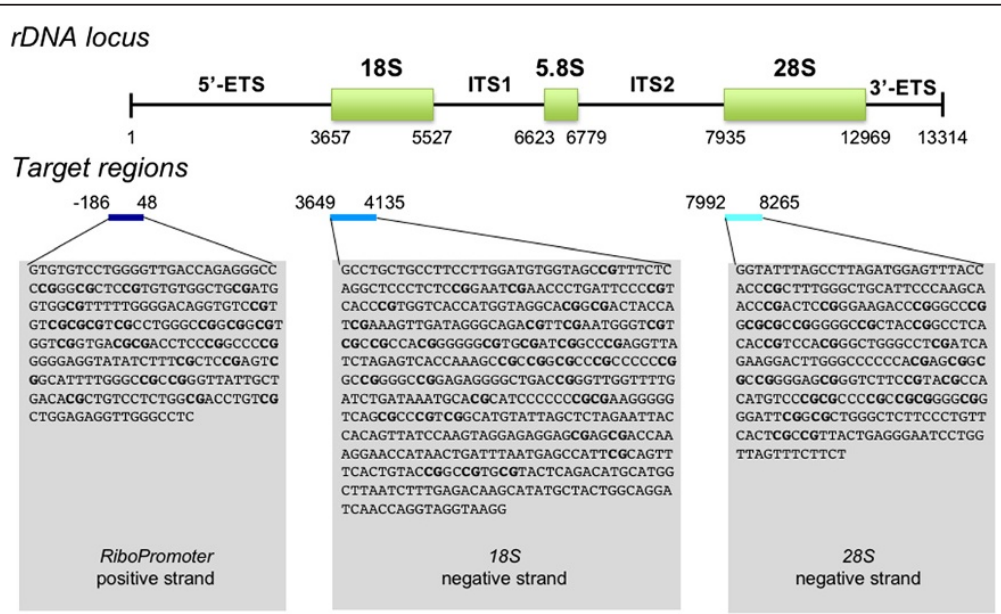

Figure 1 Location of the target regions selected for DNA methylation analysis within the rDNA locus. The picture reports a schematic representation of the rDNA locus and the location of the 3 target regions (RiboPromoter, 185 and 285) that are amplified and analyzed by the MassARRAY EpiTYPER assay. Base positions are relative to the transcription starting site $(+1)$ of rRNA primary transcript. For each amplicon the amplified strand is indicated, together with the sequence of the unconverted target region. The CpG sites whose methylation status can be assessed by the MassARRAY EpiTYPER assay are reported in bold. Abbreviations: $5^{\prime}$-ETS, $5^{\prime}$ external transcribed spacer; ITS1, Internal transcribed spacer 1; ITS2, Internal transcribed spacer 2; 3'-ETS, 3' External transcribed spacer. 

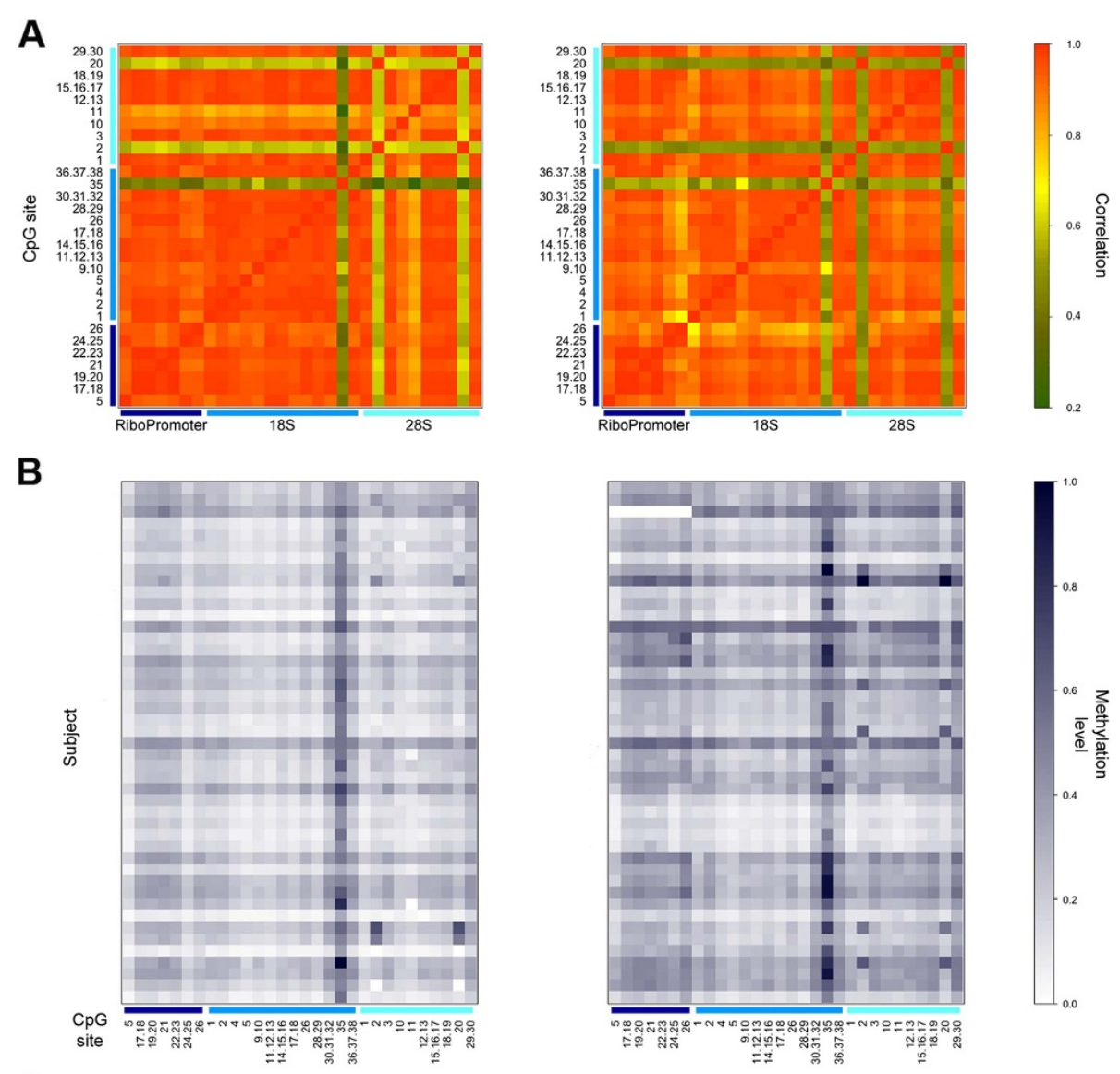

C

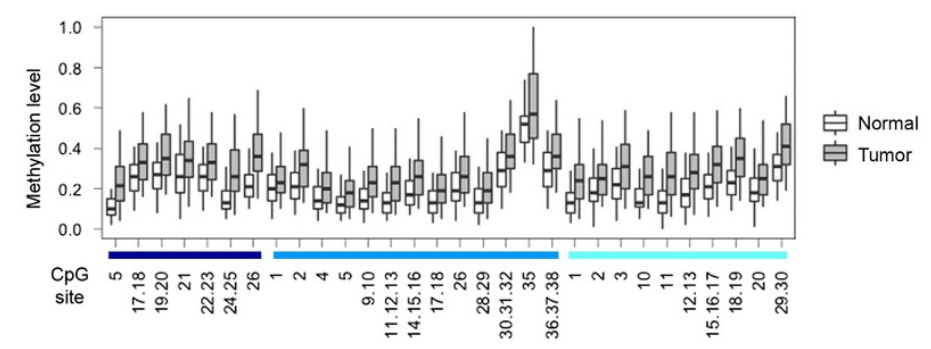

Figure 2 DNA methylation of rDNA locus in pair-matched normal and tumor tissues. (A) The correlation matrices of CpG sites analyzed in the 3 target regions (RiboPromoter, 185 and 28S) are reported for normal (left panel) and tumor (right panel) tissues. (B) The methylation levels of rDNA CpG sites are reported for 45 pair-matched normal (left panel) and tumor (right panel) tissues. (C) The boxplot compares, for each CpG site included in the analysis, the DNA methylation levels in 45 normal and tumor tissues.

CpG methylation values for each available normal-tumor tissue pair are graphically represented in Figure 2B. Considerable inter-individual variation was observed for each CpG unit both in normal and in tumor tissues. Despite this variability, we found highly significant hypermethylation in tumor in respect to matched normal breast tissue for all the analyzed CpGs (paired t-test, p-value ranging from $7.97 \times 10^{-12}$ to 0.017 depending on the $\mathrm{CpG}$ unit; Figure 2C). Comparable significant hypermethylation of rDNA regions in tumors was evident also in the 22 samples with missing normal matched tissue (Additional file 1: Figure S1).
Relationship between rDNA methylation and clinical and pathological parameters

We recovered data on 66/68 tumors deeply characterized for clinical and pathological features (listed in Table 1) and, first of all, we investigated whether there was an association between this dataset and rDNA methylation profiles. Tumor samples were classified based on patient's age, tumor histotype, size, grade (G), nuclear grade (NG), p53 status, ER and PR expression and proliferative index (Ki67) as indicated in Materials and Methods. We did not find significant differences in methylation status of rDNA between classes for investigated parameters, 
Table 1 Characteristics of the patients involved in the study Patient characteristics Age (years) (mean \pm SD): $65.26 \pm 12.68$

Age at diagnosis:

$\leq 50$

$>50$

Histotype:

IDC

DC

ILC

IDLC

Undetermined

Diameter $(\mathrm{mm})($ mean \pm SD): $18.90 \pm 9.24$

$\leq 20$

$>20$

Undetermined

Tumor size (pT classification):

$\mathrm{T} 1$

T2

T3

Undetermined

Tumor grade (G):

G1

G2

G3

Undetermined

Nucleolar size $(\mu \mathrm{m} 2)$ :

$\leq 5$

$>5$

Undetermined

p53 expression (positivity):

$\leq 10$ (p53 wild type)

$>10$ (mutated p53)

Undetermined

ER expression:

Negative

Positive

Undetermined

PR expression:

Negative

Positive

Undetermined

Ki67 expression:

$\geq 20$

$<20$

Undetermined except for NG (Table 2). Indeed, a general trend towards rDNA hypermethylation was observed in NG = 3 samples respect to $\mathrm{NG}=1$ and $\mathrm{NG}=2$ samples (Figure $3 \mathrm{~A}$ ), with statistically significant differences (ANOVA analysis) for several CpG units within RiboPromoter; $18 S$ and $28 S$ (Table 2).

There is evidence that the quantitative distribution of the nucleolar organizer regions (NORs) after their select-
55

2

8

\section{1}

2 ive staining with silver is closely related to the rates of rRNA transcription and of ribosome biogenesis, thus representing a morphological parameter of the rate of ribosome biogenesis [24-26]. We therefore focused on evaluating the relationship between the rate of ribosome biogenesis estimated by measuring the nucleolar size after selective silver staining of NORs and rDNA methylation. We successfully silver stained and measured 64/68 breast tissue specimens (Figure 3B). In order to compare the rDNA methylation levels respect to nucleolar size, we divided samples into two groups on the basis of this parameter: 41 samples showed a nucleolar area $\leq 5 \mu \mathrm{m}^{2}$, whilst for 23 samples it was more than 5 . We found that several CpG units within RiboPromoter, $18 S$ and $28 S$ rDNA regions were differently methylated between the two groups (Table 2, Figure 3C). For all CpG units we found that lower levels of rDNA methylation were associated to a higher rate of ribosome biogenesis (Figure 3C).

Considering that one of the parameters influencing nuclear grade classification is the presence of a prominent nucleolus, the results on nucleolar size appear in conflict with those on nuclear grade, being the average methylation of several sites of rDNA higher in NG3 tumors. To clarify this issue we evaluated the relationship between nuclear grade, nucleolar size and rDNA methylation. As previously observed $[27,28]$, a nucleolar area $>5$ occurred more frequently in samples with $\mathrm{NG}=3$ than in samples with $\mathrm{NG}=1$ o $\mathrm{NG}=2$ (Figure $4 \mathrm{~A}$; chi-squared test $\mathrm{p}$-value $=0.048$ ). Interestingly in $\mathrm{NG}=1$ and $\mathrm{NG}=2$ samples rDNA methylation levels were not significantly related to nucleolar size, while most of the CpG units resulted hypermethylated when a NG $=3$ co-occurred with nucleolar size $\leq 5 \mu \mathrm{m}^{2}$ (Figure $4 \mathrm{~B}$ ).

\section{Relationship between nucleolar size and rDNA methylation differences in tumor-normal tissue pairs}

Finally, we investigated whether irrespectively to the absolute value of rDNA methylation in tumor tissue, the extent of rDNA hypermethylation in tumor compared to normal matched tissue could be related to nucleolar size. To this purpose, for each CpG unit we calculated DNA methylation difference between tumor and matched normal tissue and performed hierarchical clustering analysis on these differences values (Figure 5A). Hierarchical clustering classified the 45 samples in 2 groups (indicated as 
Table 2 ANOVA test of association between rDNA methylation and clinical and pathological parameters

\begin{tabular}{|c|c|c|c|c|c|c|c|c|c|c|c|}
\hline CpG unit & Age & Histotype & Grade & Nuclear grade & Diameter & pT & p53 & ER & PR & Ki67 & Nucleolar size \\
\hline Ribo_CpG_5 & 0.74 & 0.16 & 0.37 & 0.02 & 0.89 & 0.93 & 0.57 & 0.67 & 0.96 & 0.58 & 0.04 \\
\hline Ribo_CpG_17.18 & 0.70 & 0.12 & 0.28 & 0.08 & 0.70 & 0.74 & 0.79 & 0.62 & 0.91 & 1.00 & 0.09 \\
\hline Ribo_CpG_19.20 & 0.65 & 0.11 & 0.52 & 0.16 & 0.67 & 0.67 & 0.54 & 0.91 & 0.83 & 0.74 & 0.04 \\
\hline Ribo_CpG_21 & 0.72 & 0.09 & 0.33 & 0.13 & 0.61 & 0.59 & 0.55 & 0.92 & 0.73 & 0.77 & 0.05 \\
\hline Ribo_CpG_22.23 & 0.70 & 0.12 & 0.28 & 0.08 & 0.70 & 0.74 & 0.79 & 0.62 & 0.91 & 1.00 & 0.09 \\
\hline Ribo_CpG_24.25 & 0.92 & 0.20 & 0.38 & 0.04 & 0.55 & 0.75 & 0.57 & 0.95 & 0.80 & 0.88 & 0.02 \\
\hline Ribo_CpG_26 & 0.96 & 0.26 & 0.73 & 0.26 & 0.60 & 0.67 & 0.31 & 0.52 & 0.42 & 0.46 & 0.01 \\
\hline X18S_CpG_1 & 0.59 & 0.07 & 0.45 & 0.18 & 0.82 & 0.73 & 0.34 & 0.74 & 0.91 & 0.87 & 0.21 \\
\hline X18S_CpG_2 & 0.87 & 0.07 & 0.47 & 0.14 & 0.84 & 0.87 & 0.43 & 0.93 & 0.82 & 0.82 & 0.06 \\
\hline X18S_CpG_4 & 0.52 & 0.06 & 0.40 & 0.05 & 0.97 & 0.85 & 0.60 & 0.58 & 0.88 & 0.67 & 0.14 \\
\hline X18S_CpG_5 & 0.63 & 0.13 & 0.39 & 0.04 & 0.94 & 0.94 & 0.51 & 0.76 & 0.99 & 0.39 & 0.15 \\
\hline X18S_CpG_9.10 & 0.86 & 0.47 & 0.25 & 0.04 & 0.51 & 0.54 & 0.46 & 0.96 & 0.81 & 0.85 & 0.05 \\
\hline X18S_CpG_11.12.13 & 0.57 & 0.09 & 0.17 & 0.01 & 0.64 & 0.85 & 0.90 & 0.52 & 0.75 & 0.44 & 0.08 \\
\hline X18S_CpG_14.15.16 & 0.62 & 0.13 & 0.21 & 0.02 & 0.96 & 0.99 & 0.83 & 0.54 & 0.81 & 0.32 & 0.20 \\
\hline X18S_CpG_17.18 & 0.45 & 0.21 & 0.34 & 0.03 & 0.87 & 0.82 & 0.76 & 0.61 & 0.90 & 0.42 & 0.18 \\
\hline X18S_CpG_26 & 0.53 & 0.09 & 0.45 & 0.13 & 0.91 & 0.72 & 0.73 & 0.56 & 0.86 & 0.86 & 0.20 \\
\hline X18S_CpG_28.29 & 0.54 & 0.15 & 0.14 & 0.01 & 0.87 & 0.80 & 0.80 & 0.21 & 0.38 & 0.27 & 0.24 \\
\hline X18S_CpG_30.31.32 & 0.76 & 0.21 & 0.22 & 0.05 & 0.73 & 0.79 & 0.85 & 0.39 & 0.64 & 0.72 & 0.12 \\
\hline X18S_CpG_35 & 0.92 & 0.85 & 0.99 & 0.96 & 0.25 & 0.26 & 0.21 & 0.39 & 0.39 & 0.83 & 0.25 \\
\hline X18S_CpG_36.37.38 & 0.76 & 0.21 & 0.22 & 0.05 & 0.73 & 0.79 & 0.85 & 0.39 & 0.64 & 0.72 & 0.12 \\
\hline X28S_CpG_1 & 0.57 & 0.12 & 0.29 & 0.02 & 0.78 & 0.90 & 0.64 & 0.80 & 0.95 & 0.56 & 0.06 \\
\hline X28S_CpG_2 & 0.78 & 0.63 & 0.62 & 0.86 & 0.79 & 0.73 & 0.19 & 0.14 & 0.19 & 0.16 & 0.10 \\
\hline X28S_CpG_3 & 0.41 & 0.13 & 0.45 & 0.13 & 0.79 & 0.81 & 0.81 & 0.55 & 0.82 & 0.79 & 0.14 \\
\hline X28S_CpG_10 & 0.83 & 0.11 & 0.44 & 0.08 & 0.54 & 0.65 & 0.65 & 0.88 & 0.95 & 0.89 & 0.07 \\
\hline X28S_CpG_11 & 0.81 & 0.25 & 0.40 & 0.06 & 0.93 & 1.00 & 0.84 & 0.81 & 0.99 & 0.94 & 0.08 \\
\hline X28S_CpG_12.13 & 0.74 & 0.13 & 0.46 & 0.08 & 0.97 & 0.89 & 0.40 & 0.91 & 0.60 & 0.82 & 0.05 \\
\hline X28S_CpG_15.16.17 & 0.60 & 0.21 & 0.33 & 0.03 & 0.82 & 0.95 & 0.67 & 0.79 & 0.98 & 0.81 & 0.08 \\
\hline X28S_CpG_18.19 & 0.75 & 0.17 & 0.46 & 0.08 & 0.84 & 0.83 & 0.52 & 0.95 & 0.80 & 0.97 & 0.03 \\
\hline X28S_CpG_20 & 0.78 & 0.63 & 0.62 & 0.86 & 0.79 & 0.73 & 0.19 & 0.14 & 0.19 & 0.16 & 0.10 \\
\hline X28S_CpG_29.30 & 0.75 & 0.16 & 0.42 & 0.08 & 0.74 & 0.86 & 0.46 & 0.79 & 0.62 & 0.78 & 0.04 \\
\hline
\end{tabular}

p-values less than 0.05 are reported in bold.

$\mathrm{A}$ and $\mathrm{B}$ ) ranging from low to marked rDNA hypermethylation of tumor tissue. Interestingly, group B comprised samples with very limited or absent rDNA hypermethylation in tumor tissue, indicating that increased rDNA methylation is not a feature shared by all breast carcinomas. Subsequently, we investigated if nucleolar areas were different in the 2 groups resulting from hierarchical clustering. ANOVA analysis showed that nucleolar size was significantly higher in group B samples (smaller DNA methylation difference in tumor-normal tissue pair, i.e., lower hypermethylation in tumor samples) respect to group A samples (higher DNA methylation difference in tumor-normal tissue pair, i.e. stronger hypermethylation in tumor samples) ( $p$-value $=0.006$; Figure 5B). Similar results were achieved when only $\mathrm{NG}=3$ samples were considered (Additional file 2: Figure S2). No statistically significant differences were observed between group A and group $\mathrm{B}$ when the other clinical and pathological parameters were considered.

\section{Discussion}

DNA methylation is a key regulator of gene expression and of genome architecture, and defects in its regulation often occur in several human diseases, including cancer. As many other types of tumors, up to $50 \%$ of cases of breast cancer show hypomethylation of repetitive DNA sequences and transposable elements, which substantially contributes to genomic instability [29]. Moreover, genome-wide studies on tumor tissues and breast cancer cell lines have reported aberrant hypermethylation of the 

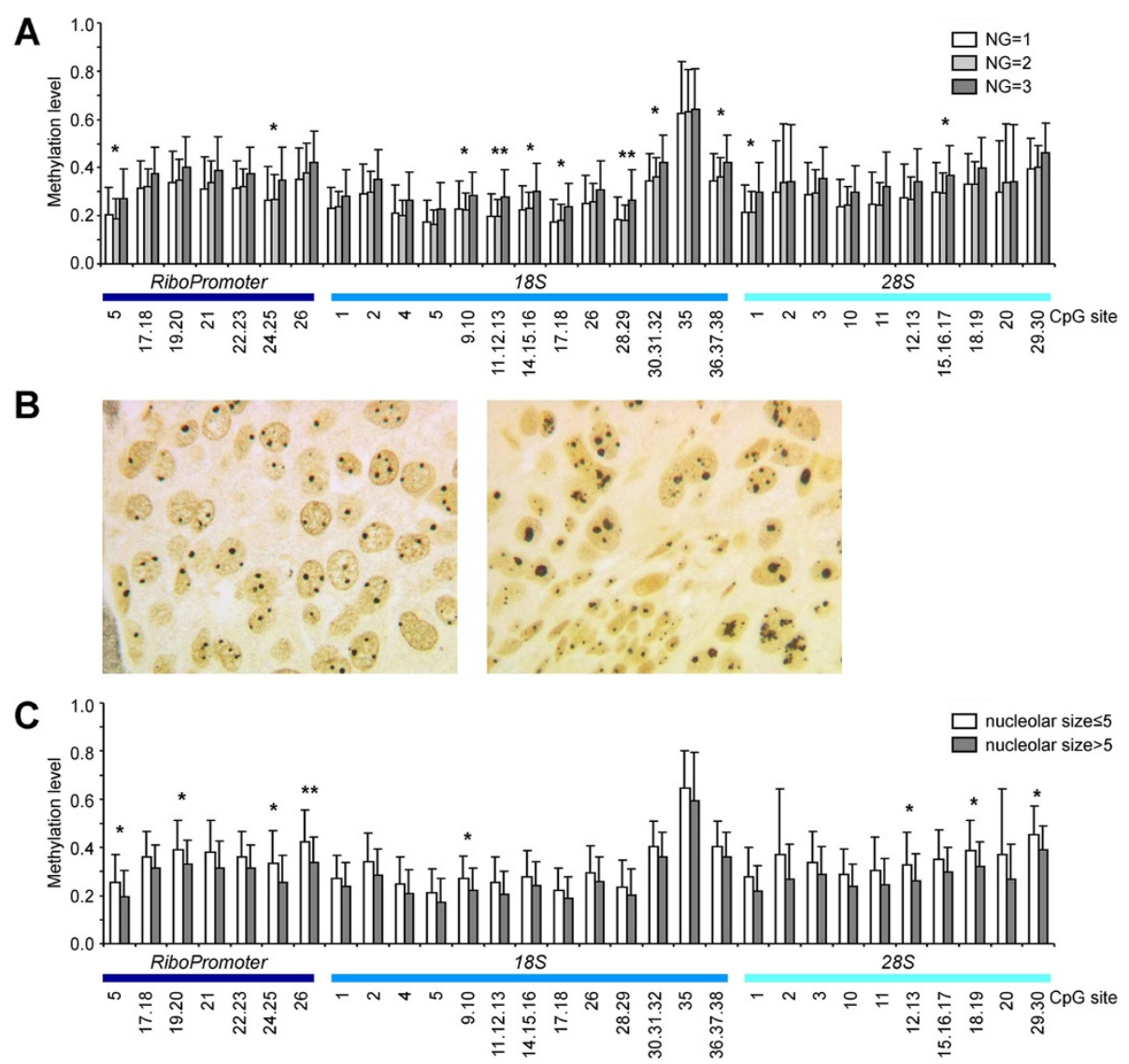

Figure 3 Relationship between rDNA methylation and tumor parameters. (A) Mean methylation levels of rDNA CpG sites in tumor samples divided for nuclear grade (NG). Standard deviation bars are reported. (B) Silver staining of two breast carcinomas. Note the higher quantity of silver stained nucleolar structures in left panel compared with those in right panel. (C) Mean methylation levels of rDNA CpG sites in tumor samples divided for nucleolar size values. Standard deviation bars are reported.

CpG islands of several genes, including tumor suppressors [30-33].

In this study, we specifically analyzed the methylation of rDNA genes in breast cancer tissues. Indeed, altered regulation of ribosome biogenesis is a common feature of many cancers, and it has been deeply investigated in breast tumors [34]. In proliferating cancer cells, the rapidity of cell proliferation is strictly dependent on ribosome production $[24,25,35]$. This is one of the major factors contributing to the growth rate of a tumor mass inside the host, which is one of the most important prognostic factors in oncology. In human carcinomas the association of nucleolar hypertrophy with bad prognoses is noteworthy and there is an increasing amount of data that suggests an active role of the nucleolus in tumorigenesis $[3,36]$. In line with this, ribosome synthesis has been also identified as a promising target for antineoplastic therapy [37-44].

The methylation status of rDNA promoter, which is CpG-rich in human [45-47], was investigated in breast cancer tissues respect to matched normal tissues. The methylation of two CpG-rich regions located at the 5' of $18 \mathrm{~S}$ and $28 \mathrm{~S}$ sequences was considered too. Our data indicate an increased rDNA methylation in tumors compared to normal tissues. Although this finding is unexpected, as neoplastic transformation should sustain ribosome biogenesis and therefore rDNA hypomethylation, similar results have been previously described. Yan and colleagues showed increased rDNA methylation levels in breast cancer biopsies compared to normal control tissue and found that rDNA hypermethylation was associated with the oestrogen receptor negative and with moderately or poorly differentiated tumors [19]. The technical approach employed in this work provided information about overall methylation status of rDNA, regardless to the epigenetic regulation of specific CpG sites [19]. Our results confirmed rDNA hypermethylation in breast tumors using the MassARRAY EpiTYPER assay, a technique that allows to assess methylation levels with single base resolution and that is more sensitive and quantitative compared to Southern blot and to clonal-sequencing of bisulfitetreated DNA. This approach allowed us to deeply 

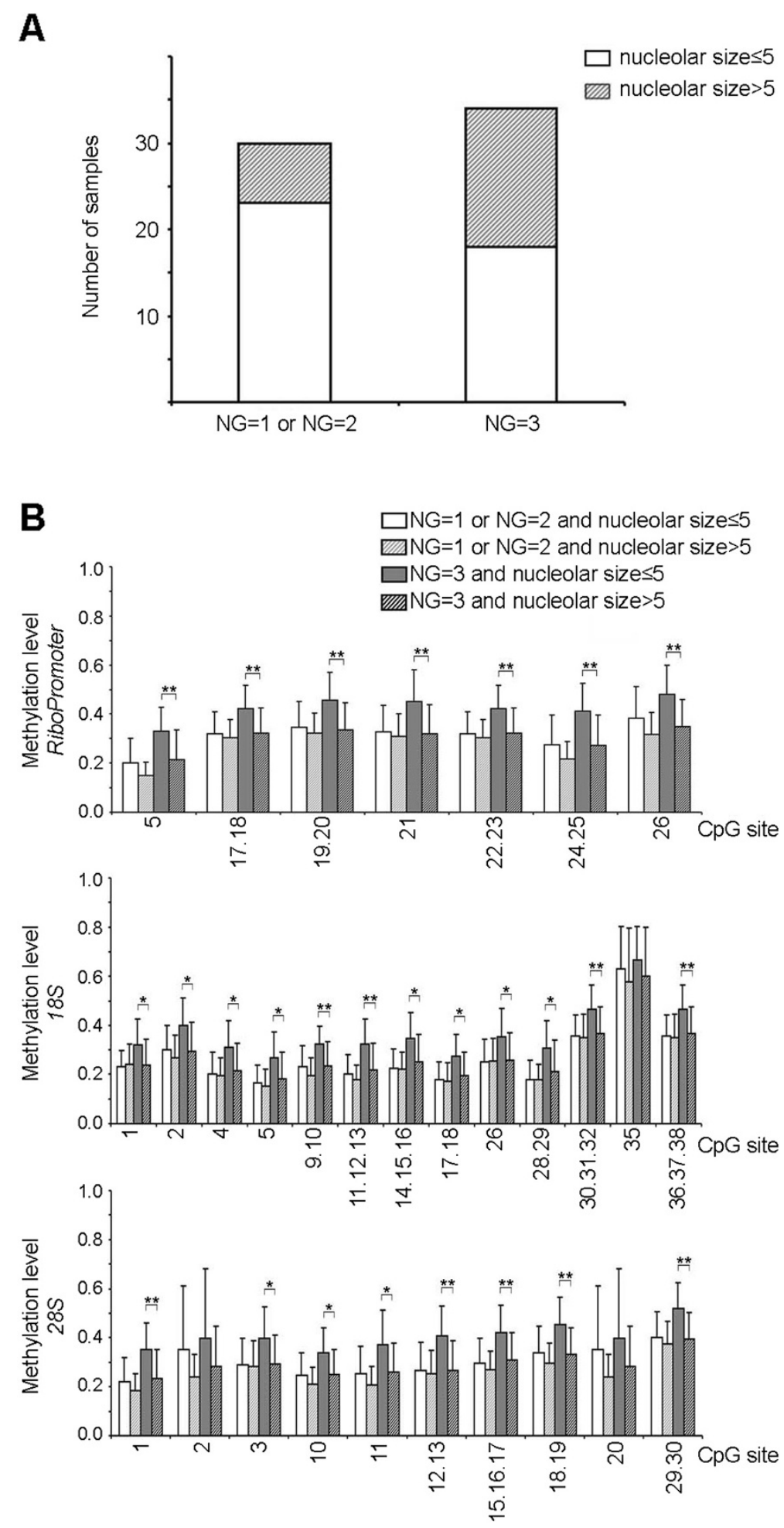

Figure 4 Relationship between nuclear grade, nucleolar size and rDNA methylation. (A) Classification of the analyzed breast carcinoma samples depending on NG and nucleolar size values. (B) Mean methylation levels of rDNA CpG sites in tumor samples divided in four classes depending on NG and nucleolar size values. Standard deviation bars are reported. 


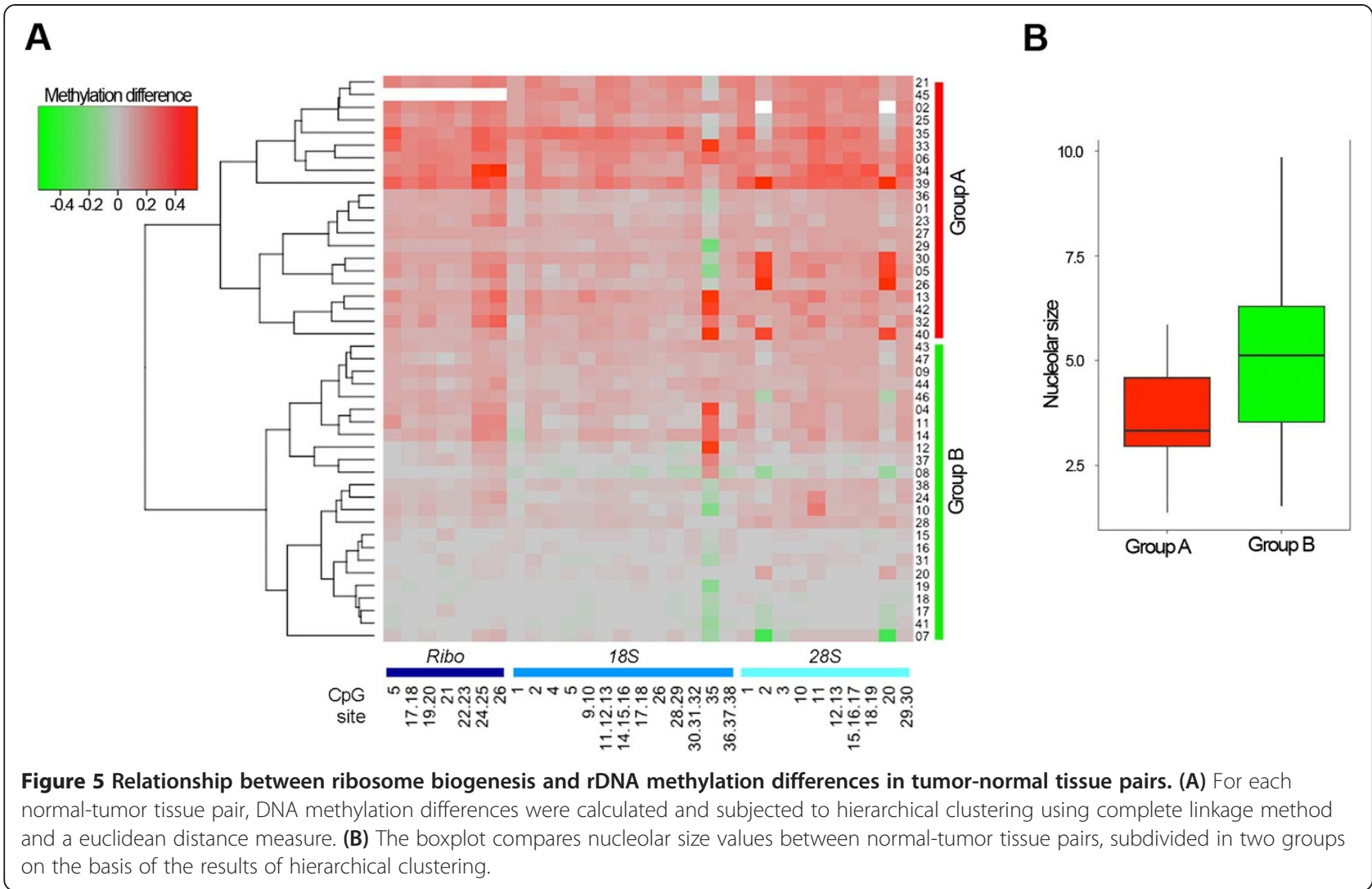

characterize DNA methylation profile of rDNA. As expected, in normal tissues the CpG sites in the promoter showed a strong correlation in their methylation levels. Moreover, also the methylation status of the $\mathrm{CpG}$ sites within the gene body ( $18 \mathrm{~S}$ and $28 \mathrm{~S}$ regions) resulted highly correlated, suggesting a tight control over the entire region in normal conditions. Correlation levels were slightly but significantly lower in tumor samples respect to normal controls, indicating that a loss in the epigenetic control, which is a common characteristic of cancer, occurs also in rDNA region.

Surprisingly, rDNA methylation of normal breast tissues showed substantial inter-individual variation, ranging from $20 \%$ to $40 \%$ depending on the $\mathrm{CpG}$ site. The biological basis of this strong variability is not clear, although it should be considered that ribosomal biogenesis, and potentially also rDNA methylation, is strongly affected by environmental factors, such as the intracellular energy status [48].

Yan and coworkers showed that higher rDNA methylation levels in tumor breast tissues were correlated with ER-negativity and suggested that they could be predictive of the tumor propensity to hypermethylate ER promoter. We did not find any significant association between rDNA methylation and ER status, but it should be considered that in our cohort ER negative cases were only a minor part of the samples (11/68 compared to $27 / 58$ in the work by Yan [19]). On the contrary, significant association was found between methylation values of several sites of rDNA loci and NG and nucleolar size values. Although only some CpGs reached statistical significance, the entire locus showed the same trend in terms of DNA methylation variations, confirming a common regulation of the $\mathrm{CpG}$ sites within the region. The nucleolar size evaluation after its selective staining with silver is a well established method used in tumor pathology for tumor characterization, being nucleolar hypertrophy associated with bad prognosis. Together with nuclear polymorphism, the presence of prominent nucleoli is one of the parameters influencing NG classification. We observed that a subgroup of samples with NG $=3$ but nucleolar size $\leq 5 \mu \mathrm{m}^{2}$ showed higher rDNA methylation levels, suggesting that in breast tumors the methylation status of rDNA loci can affect the rate of ribosome biogenesis and somehow counteract other adverse pathological conditions. Accordingly, we identified a subgroup of patients in which the presence of large nucleoli was associated to limited or absent rDNA hypermethylation of tumor tissue respect to matched normal control. In these tumors the lack of rDNA hypermethylation could represent an important factor to cope with the need for a particularly intense biosynthetic activity. In addition, this observation confirms 
an epigenetic regulation of ribosomal biogenesis in breast cancer and indicates that the rate of rDNA hypermethylation can significantly differ between patients. Importantly, samples showing small tumor-normal tissues differences had higher nucleolar size, indicating that not only the rDNA methylation level, but also the extent of rDNA hypermethylation in respect to normal tissue could represent a marker of breast cancer progression and, in principle, could be explored as a potential prognostic marker for this tumor type.

rDNA hypermethylation was described in other women's cancers, including ovarian cancer [49] and endometrial carcinoma [50]. The mechanisms and the dynamics that lead to rDNA hypermethylation in these tumors are not clear, also because tumor progression should in theory sustain higher levels or ribosome biogenesis, and therefore rDNA hypomethylation in respect to normal tissue. In all the cases, higher levels of rDNA methylation were associated to better prognosis and longer disease-free and overall survival, suggesting that rDNA methylation could have a role in the biological and clinical behavior of the tumors. One intriguing scenario is that rDNA hypermethylation may be a defense response against tumor progression, but further analyses are needed to explore this issue. With respect to previous studies, where the relationship between rDNA methylation and ribosomal biogenesis was not considered, we demonstrated for the first time that rDNA methylation is associated to nucleolar size in breast cancer. Future studies should assess if rDNA methylation affects the rate of rRNA transcription and therefore the proliferative potential of tumor cells.

\section{Conclusions}

In conclusion, in this study we showed that i) the methylation status of the $\mathrm{CpG}$ sites within the rDNA promoter and the $5^{\prime}$ of $18 \mathrm{~S}$ and $28 \mathrm{~S}$ sequences is tightly co-regulated in normal breast tissue, while in tumor tissue it is slightly but significantly lower; ii) rDNA methylation tends to be higher in breast cancer tissues respect to normal counterpart; iii) rDNA methylation levels are associated to NG and nucleolar size values and iv) in a subgroup of patients larger nucleolar size is associated with limited rDNA hypermethylation in tumor respect to matched normal tissue.

\section{Additional files}

Additional file 1: Supplementary Figure 1. DNA methylation of rDNA locus in normal and unrelated tumor tissues. The boxplot compares, for each CPG site included in the analysis, the DNA methylation levels in 45 normal tissues and 23 unrelated tumor samples.

Additional file 2: Supplementary Figure 2. Relationship between ribosome biogenesis and rDNA methylation differences in tumor-normal tissue pairs having $N G=3$. (A) Only breast carcinomas with $N G=3$ were considered. For each normal-tumor tissue pair, DNA methylation differences were calculated and subjected to hierarchical clustering. (B) The boxplot compares nucleolar size values between normal-tumor tissue pairs, subdivided in two groups on the basis of the results of hierarchical clustering

\section{Abbreviations}

rDNA: Ribosomal DNA; rRNA: Ribosomal RNA; TSS: Transcription starting site; UCE: Upstream control element; G: Tumor grade; NG: Nuclear grade; AgNORs: Silver-stained Nucleolar Organizer regions.

\section{Competing interests}

The authors declare that they have no competing interests.

\section{Authors' contributions}

LM, PG, SS, CF, MGB and AP conceived the study and wrote the article. MGB and $A P$ developed the methodology. MGB, AP, MP, DT, CG, CP carried out the experimentation, acquired the data and performed statistical analysis. All authors read and approved the final manuscript for publication.

\section{Acknowledgements}

We thank Vilma Mantovani and Elena Marasco for their technical support during experimental procedure of DNA methylation analysis at CRBA (Applied Biomedical Research Center, S. Orsola-Malpighi Polyclinic, Bologna, Italy). This work was supported by grants from the Italian Association for Cancer Research (IG-11416) to L. Montanaro and from European Union's Seventh Framework Programme (IDEAL project, 259679) to C. Franceschi.

\section{Author details}

${ }^{1}$ Department of Experimental, Diagnostic and Specialty Medicine, University of Bologna, Bologna, Italy. ${ }^{2}$ Personal Genomics S.r.l., Verona, Italy.

${ }^{3}$ Interdepartmental Center "L. Galvani", University of Bologna, Bologna, Italy. ${ }^{4}$ Centro Interdipartimentale di Ricerche sul Cancro 'Giorgio Prodi'-CIRC, University of Bologna, Bologna, Italy. ${ }^{5}$ Department of Biological, Geological and Environmental Sciences, University of Bologna, Bologna, Italy. ${ }^{6}$ Applied Biomedical Research Center, S. Orsola-Malpighi Polyclinic, Bologna, Italy.

Received: 27 December 2013 Accepted: 30 April 2014

Published: 22 May 2014

\section{References}

1. Kopp K, Gasiorowski JZ, Chen D, Gilmore R, Norton JT, Wang C, Leary DJ, Chan EKL, Dean DA, Huang S: Pol I transcription and pre-rRNA processing are coordinated in a transcription-dependent manner in mammalian cells. Mol Biol Cell 2007, 18:394-403.

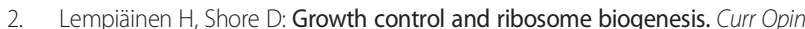
Cell Biol 2009, 21:855-863.

3. Montanaro L, Treré D, Derenzini M: Nucleolus, ribosomes, and cancer. Am J Pathol 2008, 173:301-310.

4. Schlesinger S, Selig S, Bergman Y, Cedar H: Allelic inactivation of rDNA loci. Genes Dev 2009, 23:2437-2447.

5. Santoro R, Li J, Grummt I: The nucleolar remodeling complex NoRC mediates heterochromatin formation and silencing of ribosomal gene transcription. Nat Genet 2002, 32:393-396.

6. Tan BC-M, Yang C-C, Hsieh C-L, Chou Y-H, Zhong C-Z, Yung BY-M, Liu H: Epigeneitc silencing of ribosomal RNA genes by Mybbp1a. J Biomed SCi 2012, 19:57.

7. Ghoshal K, Majumder S, Datta J, Motiwala T, Bai S, Sharma SM, Frankel W, Jacob ST: Role of human ribosomal RNA (rRNA) promoter methylation and of methyl-CpG-binding protein MBD2 in the suppression of rRNA gene expression. J Biol Chem 2004, 279:6783-6793.

8. McStay B, Grummt I: The epigenetics of rRNA genes: from molecular to chromosome biology. Annu Rev Cell Dev Biol 2008, 24:131-157.

9. Majumder S, Ghoshal K, Datta J, Smith DS, Bai S, Jacob ST: Role of DNA methyltransferases in regulation of human ribosomal RNA gene transcription. J Biol Chem 2006, 281:22062-22072.

10. Brown SE, Szyf M: Dynamic epigenetic states of ribosomal RNA promoters during the cell cycle. Cell Cycle 2008, 7:382-390.

11. Pietrzak M, Rempala G, Nelson PT, Zheng J-J, Hetman M: Epigenetic silencing of nucleolar rRNA genes in Alzheimer's disease. PLOS One 2011, 6:e22585. 
12. McGowan PO, Sasaki A, Huang TCT, Unterberger A, Suderman M, Ernst C, Meaney MJ, Turecki G, Szyf M: Promoter-wide hypermethylation of the ribosomal RNA gene promoter in the suicide brain. PLoS One 2008, 3:e2085

13. Javierre BM, Fernandez AF, Richter J, Al-Shahrour F, Martin-Subero الر, Rodriguez-Ubreva J, Berdasco M, Fraga MF, O'Hanlon TP, Rider LG, Jacinto FV, Lopez-Longo FJ, Dopazo J, Forn M, Peinado MA, Carreño L, Sawalha AH, Harley JB, Siebert R, Esteller M, Miller FW, Ballestar E: Changes in the pattern of DNA methylation associate with twin discordance in systemic lupus erythematosus. Genome Res 2010, 20:170-179.

14. Oakes CC, Smiraglia DJ, Plass C, Trasler JM, Robaire B: Aging results in hypermethylation of ribosomal DNA in sperm and liver of male rats. Proc Natl Acad Sci U S A 2003, 100:1775-1780.

15. Machwe A, Orren DK, Bohr VA: Accelerated methylation of ribosomal RNA genes during the cellular senescence of Werner syndrome fibroblasts. FASEB J 2000, 14:1715-1724.

16. Thomas G: An encore for ribosome biogenesis in the control of cell proliferation. Nat Cell Biol 2000, 2:E71-E72.

17. Schmidt EV: The role of c-myc in cellular growth control. Oncogene 1999 18:2988-2996.

18. Uemura M, Zheng Q, Koh CM, Nelson WG, Yegnasubramanian S, De Marzo AM: Overexpression of ribosomal RNA in prostate cancer is common but not linked to rDNA promoter hypomethylation. Oncogene 2012, 31:1254-1263.

19. Yan PS, Rodriguez FJ, Laux DE, Perry MR, Standiford SB, Huang TH: Hypermethylation of ribosomal DNA in human breast carcinoma. Br J Cancer 2000, 82:514-517.

20. Beahrs OH: Pretreatment staging of cancer. Cancer 1989, 64:275-278. discussion 282-284.

21. Garagnani P, Bacalini MG, Pirazzini C, Gori D, Giuliani C, Mari D, Di Blasio AM, Gentilini D, Vitale G, Collino S, Rezzi S, Castellani G, Capri M, Salvioli S, Franceschi C: Methylation of ELOVL2 gene as a new epigenetic marker of age. Aging Cell 2012, 11:1132-1134.

22. Trerè D: AgNOR staining and quantification. Micron 2000, 31:127-131.

23. Espada J, Ballestar E, Santoro R, Fraga MF, Villar-Garea A, Németh A, LopezSerra L, Ropero S, Aranda A, Orozco H, Moreno V, Juarranz A, Stockert JC, Längst G, Grummt I, Bickmore W, Esteller M: Epigenetic disruption of ribosomal RNA genes and nucleolar architecture in DNA methyltransferase 1 (Dnmt1) deficient cells. Nucleic Acids Res 2007, 35:2191-2198.

24. Derenzini M, Trerè D, Pession A, Montanaro L, Sirri V, Ochs RL: Nucleolar function and size in cancer cells. Am J Pathol 1998, 152:1291-1297.

25. Derenzini M, Trerè D, Pession A, Govoni M, Sirri V, Chieco P: Nucleolar size indicates the rapidity of cell proliferation in cancer tissues. J Pathol 2000, 191:181-186.

26. Hernandez-Verdun D: The nucleolus: a model for the organization of nuclear functions. Histochem Cell Biol 2006, 126:135-148.

27. Derenzini M, Ceccarelli C, Santini D, Taffurelli M, Treré D: The prognostic value of the AgNOR parameter in human breast cancer depends on the pRb and p53 status. J Clin Pathol 2004, 57:755-761.

28. Derenzini M, Montanaro L, Treré D: What the nucleolus says to a tumour pathologist. Histopathology 2009, 54:753-762.

29. Soares J, Pinto AE, Cunha CV, André S, Barão I, Sousa JM, Cravo M: Global DNA hypomethylation in breast carcinoma: correlation with prognostic factors and tumor progression. Cancer 1999, 85:112-118.

30. Morita S, Takahashi R-U, Yamashita R, Toyoda A, Horii T, Kimura M, Fujiyama A, Nakai K, Tajima S, Matoba R, Ochiya T, Hatada I: Genome-wide analysis of DNA methylation and expression of micrornas in breast cancer cells. Int J Mol Sci 2012, 13:8259-8272.

31. Kikuyama M, Takeshima H, Kinoshita T, Okochi-Takada E, Wakabayashi M, Akashi-Tanaka S, Ogawa T, Seto Y, Ushijima T: Development of a novel approach, the epigenome-based outlier approach, to identify tumorsuppressor genes silenced by aberrant DNA methylation. Cancer Lett 2012, 322:204-212

32. Rao X, Evans J, Chae H, Pilrose J, Kim S, Yan P, Huang R-L, Lai H-C, Lin H, Liu Y, Miller D, Rhee J-K, Huang Y-W, Gu F, Gray JW, Huang T-M, Nephew KP: CpG island shore methylation regulates caveolin-1 expression in breast cancer. Oncogene 2013, 32:4519-4528.

33. Faryna M, Konermann C, Aulmann S, Bermejo JL, Brugger M, Diederichs S, Rom J, Weichenhan D, Claus R, Rehli M, Schirmacher P, Sinn H-P, Plass C, Gerhauser C: Genome-wide methylation screen in low-grade breast cancer identifies novel epigenetically altered genes as potential biomarkers for tumor diagnosis. FASEB J 2012, 26:4937-4950
34. Belin S, Beghin A, Solano-Gonzàlez E, Bezin L, Brunet-Manquat S, Textoris J, Prats A-C, Mertani HC, Dumontet C, Diaz J-J: Dysregulation of ribosome biogenesis and translational capacity is associated with tumor progression of human breast cancer cells. PLoS One 2009, 4:e7147.

35. Derenzini M, Montanaro L, Chillà A, Tosti E, Vici M, Barbieri S, Govoni M, Mazzini G, Treré D: Key role of the achievement of an appropriate ribosomal RNA complement for G1-S phase transition in H4-II-E-C3 rat hepatoma cells. J Cell Physiol 2005, 202:483-491.

36. Montanaro $L$, Treré $D$, Derenzini M: Changes in ribosome biogenesis may induce cancer by down-regulating the cell tumor suppressor potential. Biochim Biophys Acta 1825, 2012:101-110.

37. Bywater MJ, Poortinga G, Sanij E, Hein N, Peck A, Cullinane C, Wall M, Cluse L, Drygin D, Anderes K, Huser N, Proffitt C, Bliesath J, Haddach M, Schwaebe MK, Ryckman DM, Rice WG, Schmitt C, Lowe SW, Johnstone RW, Pearson RB, McArthur GA, Hannan RD: Inhibition of RNA polymerase I as a therapeutic strategy to promote cancer-specific activation of p53. Cancer Cell 2012, 22:51-65.

38. Drygin D, Lin A, Bliesath J, Ho CB, O'Brien SE, Proffitt C, Omori M, Haddach M, Schwaebe MK, Siddiqui-Jain A, Streiner N, Quin JE, Sanij E, Bywater MJ, Hannan RD, Ryckman D, Anderes K, Rice WG: Targeting RNA polymerase I with an oral small molecule CX-5461 inhibits ribosomal RNA synthesis and solid tumor growth. Cancer Res 2011, 71:1418-1430.

39. Ruggero D: Revisiting the nucleolus: from marker to dynamic integrator of cancer signaling. Sci Signal 2012, 5:pe38.

40. Drygin D, Rice WG, Grummt I: The RNA polymerase I transcription machinery: an emerging target for the treatment of cancer. Annu Rev Pharmacol Toxicol 2010, 50:131-156.

41. Hein N, Hannan KM, George AJ, Sanij E, Hannan RD: The nucleolus: an emerging target for cancer therapy. Trends Mol Med 2013, 19:643-654.

42. Bywater MJ, Pearson RB, McArthur GA, Hannan RD: Dysregulation of the basal RNA polymerase transcription apparatus in cancer. Nat Rev Cancer 2013, 13:299-314.

43. Drygin D, O'Brien SE, Hannan RD, McArthur GA, Von Hoff DD: Targeting the nucleolus for cancer-specific activation of p53. Drug Discov Today 2014, 19:259-265.

44. Montanaro L, Treré $D$, Derenzini M: The emerging role of RNA polymerase I transcription machinery in human malignancy: a clinical perspective. Onco Targets Ther 2013, 6:909-916.

45. Worton RG, Sutherland J, Sylvester JE, Willard HF, Bodrug S, Dubé I, Duff C, Kean V, Ray PN, Schmickel RD: Human ribosomal RNA genes: orientation of the tandem array and conservation of the $5^{\prime}$ end. Science 1988, 239:64-68.

46. Dante R, Percy ME, Baldini A, Markovic VD, Miller DA, Rocchi M, Niveleau A, Miller OJ: Methylation of the $5^{\prime}$ flanking sequences of the ribosomal DNA in human cell lines and in a human-hamster hybrid cell line. J Cell Biochem 1992, 50:357-362.

47. Gonzalez IL, Wu S, Li WM, Kuo BA, Sylvester JE: Human ribosomal RNA intergenic spacer sequence. Nucleic Acids Res 1992, 20:5846.

48. Murayama A, Ohmori K, Fujimura A, Minami H, Yasuzawa-Tanaka K, Kuroda T, Oie S, Daitoku H, Okuwaki M, Nagata K, Fukamizu A, Kimura K, Shimizu T, Yanagisawa J: Epigenetic control of rDNA loci in response to intracellular energy status. Cell 2008, 133:627-639.

49. Chan MWY, Wei SH, Wen P, Wang Z, Matei DE, Liu JC, Liyanarachchi S, Brown R, Nephew KP, Yan PS, Huang TH-M: Hypermethylation of $18 \mathrm{~S}$ and $28 \mathrm{~S}$ ribosomal DNAs predicts progression-free survival in patients with ovarian cancer. Clin Cancer Res 2005, 11:7376-7383.

50. Powell MA, Mutch DG, Rader JS, Herzog TJ, Huang TH-M, Goodfellow PJ: Ribosomal DNA methylation in patients with endometrial carcinoma: an independent prognostic marker. Cancer 2002, 94:2941-2952.

doi:10.1186/1471-2407-14-361

Cite this article as: Bacalini et al:: The nucleolar size is associated to the methylation status of ribosomal DNA in breast carcinomas. BMC Cancer 2014 14:361. 